\title{
Transient Response of Thin Wire above a Layered Half-Space Using TDIE/FDTD Hybrid Method
}

\author{
Bing Wei, Qiong He, Jie Li, Ren-xian Li, and Li-xin Guo \\ Department of Physics, Xidian University, Xi'an 710071, China \\ Correspondence should be addressed to Bing Wei, weibing_xd@yahoo.com.cn
}

Received 15 July 2011; Revised 26 September 2011; Accepted 20 October 2011

Academic Editor: Hu Jun

Copyright (๑) 2012 Bing Wei et al. This is an open access article distributed under the Creative Commons Attribution License, which permits unrestricted use, distribution, and reproduction in any medium, provided the original work is properly cited.

The TDIE/FDTD hybrid method is applied to calculate the transient responses of thin wire above a lossy layered half-space. The time-domain reflection of the layered half space is computed by one-dimensional modified FDTD method. Then, transient response of thin wire induced by two excitation sources (the incident wave and reflected wave) is calculated by TDIE method. Finally numerical results are given to illustrate the feasibility and high efficiency of the presented scheme.

\section{Introduction}

Among the available literatures when analyzing transient response of thin-wire above a half-space, the scattering and radiation of thin-wire structure are generally analyzed by employing time-domain integral equation (TDIE) method [1-8] or finite element method (FEM) [9], and the influence of half-space is often considered by introducing the imaging principle, as well as reflection coefficient [3]. In 1980, Parviz Parhami et al. [10] derived the general integral equation for an arbitrarily shaped thin-wire antenna over a lossy halfspace, and method of moment (MoM) in frequency domain is employed to solve the equation numerically. In his paper, far-field radiation patterns of center-fed horizontal dipole, center-fed vertical dipole, and center-fed inverted dipole are given. In 1998, Poljak established space-time integral equation of Hallen's type to deal with a straight thin wire horizontally placed above a dissipative half-space. The influence of a lossy half-space is taken into account by the Fresnel space-time reflection coefficient which appears inside the IE kernel [4]. In 2004, he calculated the transient responses of nonlinear loaded wire antenna in half-space with spatialtime Hallen equation combined with reflection coefficient method [5]. Recently, Haddad et al. used complex-time Green's functions to obtain the transient response of thinwire structures located above half-space [6].
The above literature deals only with the interactions between thin wire and homogeneous or one-layer halfspace. In many practical cases, the half-space is composed of complex dielectrics, which is usually in the form of layers. In this paper, a hybrid method that combines TDIE with FDTD is employed to study the transient responses of thin conducting wire above a lossy and layered half-space. It is well known that TDIE method is suited to simulate the scattering or radiation from thin-wire structures, whereas FDTD is a powerful tool that could model the interactions of EM waves with inhomogeneous media [11-29]. Huang et al. used the FDTD/MoM hybrid technique for modeling the radiation field of complex antennas above the heterogeneous grounds [26]. Monorchio et al. used the hybrid time-domain technique that combines the finite element, finite difference, and method of moment techniques to compute the radiation field of a thin-wire antenna near inhomogeneous dielectric bodies [27]. Hybrid method in [26, 27] uses iterationbased technique to couple 3-D FDTD(/FETD) and TDIE. The hybrid method in this paper is connected with onereflection field (neglecting higher order reflections when the distance of the wire and the interface of the layered half-space are far enough for the problems we interested) without any iteration-based procedure to consider the multiple interactions between wire structure and underlying layered half-space. To calculate first-order reflected field, only 


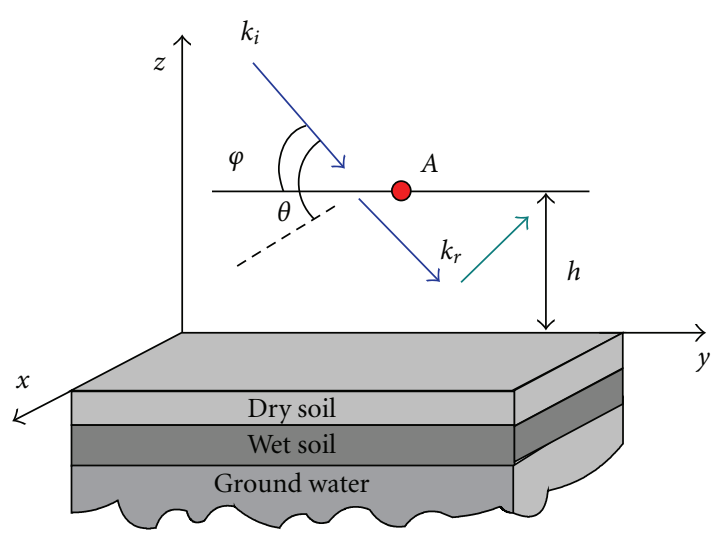

FIGURE 1: The geometry of a thin wire above layered half-space.

1-D FDTD is needed. In this case the original problem is decomposed into two subregions. The first sub-region is single-wire structure in free space, while the second subregion is the layered dielectric without any wire structure above it. Then transient response on the wire is analyzed using TDIE $[1,2]$, which has two exciting terms in the formulation, both the original incidence and the reflected wave. This hybrid algorithm utilizes advantages of both TDIE and FDTD, respectively, and furthermore no iteration is needed. Numerical examples show that this hybrid method is a very efficient way to study transient responses of wire structures above a layered half-space.

\section{Basic Theory of Hybrid Method}

The geometry of a straight thin conducting wire above multilayer dielectric media is shown in Figure 1. The thin wire is parallel to the $y$ axis, the length of wire is $l$, and the distance between the wire and interface is $h$. The angle between the incident plane and the surface of the half-space is $\theta$. The angle between the incident wave and the thin wire is $\varphi$. In this paper, the plane of incidence is determined by the incident vector $\overrightarrow{k_{i}}$ and the direction of the thin wire.

When one analyzes the transient response of the wire in Figure 1, the excitation sources include three parts: (1) the original incident wave (zero-order), (2) the reflected wave reflected by layered half-space from the original incident wave (first-reflection), and (3) the radiated field from the induced current which is reflected by the half-space and becomes incident upon the wire again (second- and higherorder reflections).

The radiation field of thin wire can be regarded as superposition of the radiation field of many electric dipoles which are parallel to the interface. Using the spherical coordinate system, the radiation electric field of an electric dipole can be written as [30]

$$
\begin{aligned}
\vec{E}=-i \omega I l \frac{\exp (i k r)}{4 \pi r} & \left\{\widehat{r}\left(\frac{i}{k r}+\left(\frac{i}{k r}\right)^{2}\right) 2 \cos \theta^{\prime}\right. \\
& \left.+\widehat{\theta^{\prime}}\left(1+\frac{i}{k r}+\left(\frac{i}{k r}\right)^{2}\right) \sin \theta^{\prime}\right\},
\end{aligned}
$$

where $i, \omega, I, l, k, r, \widehat{r}, \theta^{\prime}$, and $\widehat{\theta^{\prime}}$ are units of the imaginary number, circular frequency of the incident wave, electric current of the wire, length of the dipole, spatial frequency, the distance of the origin point to observation point, the orientation vector, the angle between the $\widehat{r}$ and the $\widehat{z^{\prime}}$ direction, and the unit vector of $\theta^{\prime}$ direction, respectively, as illustrated in Figure 2. Equation (1) gives the radiation field of an electric dipole, which is suitable for both near field and far field. The radiation field of the dipole toward all directions in which the field is perpendicularly incident towards the interface of layered half-space can be reflected and becomes incident upon the dipole again $\left(\theta^{\prime}=\pi / 2\right)$. In this case, (1) can be written as

$$
E_{\theta}=\omega I l \frac{\exp (i k r)}{4 \pi r}\left[\frac{1}{k r}+i\left(\frac{1}{(k r)^{2}}-1\right)\right]
$$

To make sure the existence of higher-order reflection does not ruin the hybridization of TDIE and FDTD, the electric field that is radiated by the dipole and reflected by halfspace and arriving at the dipole again is estimated. Suppose we have a one-meter-long wire composed of four hundred electric dipoles and the direction of the wire is parallel to the interface. Let $E_{0}, E_{1}$, and $E_{2}$ represent the radiation electric field of a dipole at $r_{0}=0.002 \mathrm{~m}$ (the radius of the wire), $r_{1}=0.25 \mathrm{~m}$ and $r_{2}=2.0 \mathrm{~m}$, respectively. According to (2) we know that the ratios of $E_{1}$ to $E_{0}$ and $E_{2}$ to $E_{0}$ basically do not change when the frequency varies from $1 \mathrm{MHz}$ to $20 \mathrm{GHz}$. Suppose the observation point is $j$ and the source point is $i$ $(i=1 \sim 400)$. The radiation electric fields of dipole jreflected by the ground half-space and reaching dipole $j$ again can be estimated by (2). The radiation electric fields of dipole $i$ $(i \neq j)$ reflected by the ground half-space and reaching dipole $j$ again are less than the reflection field which is radiated by the dipole $j$. So the maximum reflected fields of point $j$ on the surface of the thin wire are about

$$
\begin{aligned}
400 \times\left. E_{1}\right|_{0.25 \mathrm{~m}} & \approx 1.39 \times 10^{-3} R \times E_{0}, \\
400 \times\left. E_{1}\right|_{2 \mathrm{~m}} & \approx 2.5 \times 10^{-4} R \times E_{0},
\end{aligned}
$$

where $R<1$ is the reflection coefficient of the layered halfspace and the subscripts $0.25 \mathrm{~m}$ and $2 \mathrm{~m}$ indicated the wire is located 0.25 meter or 2.0 meters above the interface of the half-space, respectively. It is obvious that in these two cases the contribution of the radiation field reflected by the current is much smaller than original incident field. So highorder interactions between thin wire and half-space could be neglected in this paper.

When one applies the hybrid scheme to analyze the transient responses of wire structure above layered halfspace, TDIE is employed to study the above wire with both the original incident and reflected wave as the exciting source. In the hybrid formulations, considering the thinness nature of wire structure, the incident wave across the section of the conducting wire approximately has the same value, and the induced current on the wire is considered as line current. In forming the integral equation, the source point will be on the axis of the cylinder, whereas the observation point is positioned at the conductor surface. Therefore, the 


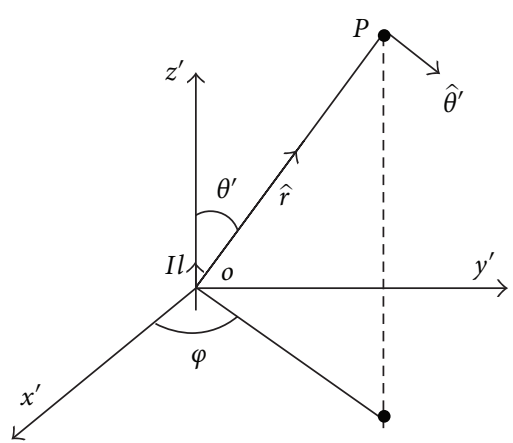

FIgURE 2: The electric dipole.

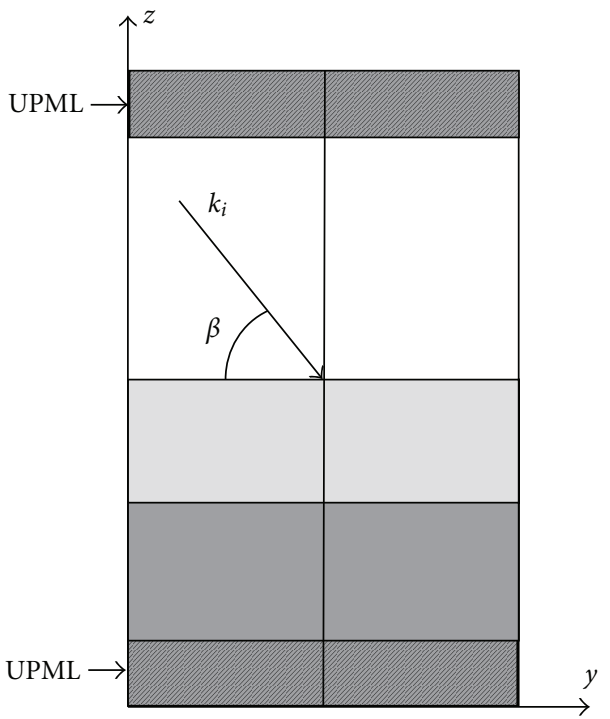

FIgUre 3: The FDTD model.

distance between the source point and observation point is always larger than or equal to the radius of the wire.

Compared with the TDIE method in free space, the TDIE method in the hybrid approach deals with two kinds of incident waves. The first is the original incident wave, which is introduced in analytical form, while the other exciting source is from the reflected wave of the underlying layered half-space, which can be calculated using one-dimensional modified FDTD method. The configuration of the half-space can be arbitrary, and the dielectric parameters can be varied layer by layer.

The FDTD model is given in Figure 3. In order to obtain the reflected wave where the conducting wire is positioned, the location of output point in the scattering field (SF) region of FDTD domain is as high as the wire, whereas the half-space is modeled in the total field region. In the implementation of FDTD, UPML is used to truncate the infinite domain and reduce numerical errors.

The general formulations of FDTD and TDIE methods will be given in the following.

2.1. FDTD Method. Suppose a straight thin wire structure is located above the layered half-space as Figure 1 shows. The electric fields have components along $y$ and $z$ axis when the model is impinged by oblique incident wave. The $y$ component can stimulate induced current whereas the $z$ component cannot.

In FDTD method, a set of finite-difference equations for the time-dependent Maxwell's curl equations system is originated by Yee [13]. These equations can be represented in a discrete form, both in space and time, employing the second-order accurate central difference formula.

Supposing the incident angle is $\beta$ (the angle of the interface of the layered half-space and the $\overrightarrow{k_{i}}$ as Figure 3 shows), the parameters of media and the field quantity are independent and denoted by $y$ and $z$, and the modified Maxwell curl equations of one-dimensional case are

$$
\begin{gathered}
-\frac{\partial E_{x 1 D}}{\partial z}=\mu \frac{\partial H_{y 1 D}}{\partial t}, \\
-\frac{1}{\sin ^{2} \beta} \frac{\partial H_{y 1 D}}{\partial z}=\varepsilon \frac{\partial E_{x 1 D}}{\partial t},
\end{gathered}
$$

where $\mu=\mu_{0} \mu_{r}$ and $\varepsilon=\varepsilon_{0} \varepsilon_{r}$ are permeability and permittivity of the media, respectively. Equations (4) and (5) can be employed to compute the reflection and transmission electricmagnetic wave in case of an oblique plane wave incident to the surface of the layered half-space.

Considering $k_{y}=k \cos \beta$ and $k_{z}=k \sin \beta$, (5) can be written as

$$
-\frac{k^{2}}{k_{z}^{2}} \frac{\partial H_{y 1 D}}{\partial z}=\varepsilon j \omega E_{x 1 D}
$$

According to the phase match theory, $k_{y}=k \cos \beta$ is a constant in each layer. Equation (6) can be written as

$$
-k^{2} \frac{\partial H_{y 1 D}}{\partial z}=\left(k^{2}-k_{y}^{2}\right) j \omega \varepsilon_{0} \varepsilon_{r} E_{x 1 D},
$$

in which $k=\omega \sqrt{\mu \varepsilon}, k_{z}^{2}=k^{2}-k_{y}^{2}$. Then we have

$$
-\frac{\partial H_{y 1 D}}{\partial z}=\left[\varepsilon_{r}-\varepsilon_{1} \cos ^{2} \beta\right] \varepsilon_{0} j \omega E_{x 1 D}
$$

Let $\varepsilon^{\prime}=\varepsilon_{r}-\varepsilon_{1} \sin ^{2} \beta$, and (8) can be written as

$$
-\frac{\partial H_{y 1 D}}{\partial z}=\varepsilon_{0} \varepsilon^{\prime} \frac{\partial E_{x 1 D}}{\partial t} .
$$

The derivatives in (4) and (9) can be approximated by using the central difference formula with the position $E_{x}(m)$ being the center point for the central difference formula in space and time instant $(n+1 / 2) \Delta t$ being the center point in time. We can get FDTD updating equation as follows:

$$
\begin{aligned}
\left.E_{x 1 D}\right|_{j} ^{n+1}= & \left.C A(m) \cdot E_{x 1 D}\right|_{j} ^{n} \\
& -C B(m) \cdot \frac{\left.H_{y 1 D}\right|_{j+1 / 2} ^{n+1 / 2}-\left.H_{y 1 D}\right|_{j-1 / 2} ^{n+1 / 2}}{\Delta z}, \\
\left.H_{y 1 D}\right|_{j+1 / 2} ^{n+1 / 2}= & \left.C P(m) \cdot H_{y 1 D}\right|_{j+1 / 2} ^{n-1 / 2} \\
& -C Q(m) \cdot\left[\frac{\left.E_{x 1 D}\right|_{j+1} ^{n}-\left.E_{x 1 D}\right|_{j} ^{n}}{\Delta z}\right],
\end{aligned}
$$




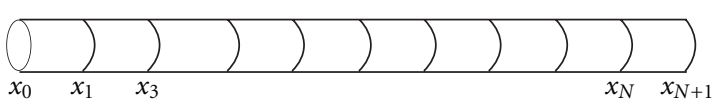

Figure 4: The thin wire is divided into $N+1$ subfield.

where $C A(m), C B(m), C P(m)$, and $C Q(m)$ are coefficient of the updating equation.

In this paper, in order to obtain the reflected wave of the layered half-space, the layered half-space is modeled in the total field (TF) region, and the output point of the reflection waves is in the scattering field (SF) region of FDTD domain. UPML absorbing conduction is used to truncate the infinite domain and reduce numerical errors.

2.2. TDIE Method. The scattering electric field of the thin wire can be expressed by the vector potential as follows:

$$
\frac{\partial \vec{E}}{\partial t}=-\frac{\partial^{2} \vec{A}}{\partial t^{2}}+c^{2} \nabla(\nabla \cdot \vec{A}) .
$$

The directed incident field $\boldsymbol{E}_{x}^{i}$ and reflected field $\boldsymbol{E}_{x}^{r}$ can be regarded as a constant across the section of wire. Applying the boundary condition for the total electric field, (11) can be written as

$$
\begin{array}{r}
\frac{\partial^{2} A_{x}}{\partial x^{2}}-\left.\frac{1}{c^{2}} \frac{\partial^{2} A_{x}}{\partial t^{2}}\right|_{r=a}=-\left.\frac{1}{c^{2}} \frac{\partial E_{x}^{i}}{\partial t}\right|_{r=a}-\left.\frac{1}{c^{2}} \frac{\partial E_{x}^{r}}{\partial t}\right|_{r=a}, \\
x \in(0, L) .
\end{array}
$$

The vector potential is given by

$$
A_{x}(x, t)=\frac{\mu_{0}}{4 \pi} \int_{x^{\prime}=0}^{L} \frac{I\left(x^{\prime}, t-\left|x-x^{\prime}\right| / c\right)}{\sqrt{\left|x-x^{\prime}\right|^{2}+a^{2}}} d x^{\prime},
$$

where $a$ and $c$ represent the radius of thin wire, and velocity of light, respectively.

The thin wire is divided into $N+1$ equal segments; the length of the segment is $\Delta x$ (as the Figure 4 shows). The basis function is defined as follows:

$$
f_{m}(x)= \begin{cases}1, & x_{m}-\frac{\Delta x}{2} \leq x \leq x_{m}+\frac{\Delta x}{2} \\ 0, & \text { otherwise. }\end{cases}
$$

Using these expansion functions, we approximate the current $I$ as follows:

$$
I(x, t) \approx \sum_{k=1}^{n} I_{k}(t) f_{k}(x)
$$

If central difference approximation is employed, (12) can be written as

$$
\begin{aligned}
\frac{A_{m+1, n}-2 A_{m, n}+A_{m-1, n}}{(\Delta x)^{2}}-\frac{A_{m, n+1}-2 A_{m, n}+A_{m, n-1}}{(c \Delta t)^{2}} \\
=-F_{m, n},
\end{aligned}
$$

where the excitation term is

$$
F_{m, n}=\frac{\partial E_{x}^{i}\left(x_{m}, t_{n}\right)}{\partial t}+\frac{\partial E_{x}^{r}\left(x_{m}, t_{n}\right)}{\partial t} .
$$

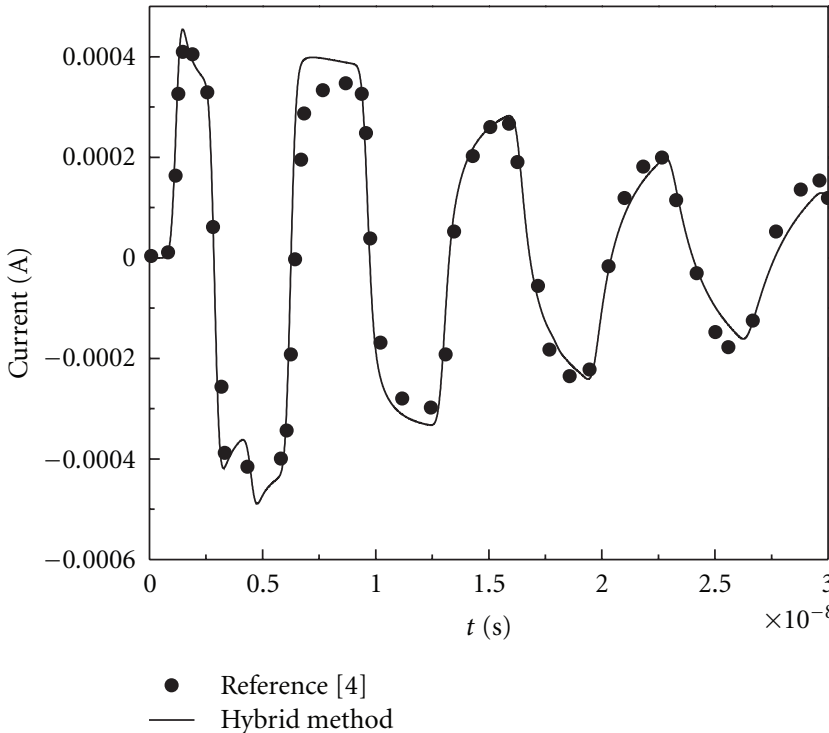

FIGURE 5: Current induced at midpoint of a straight thin wire above half-space.

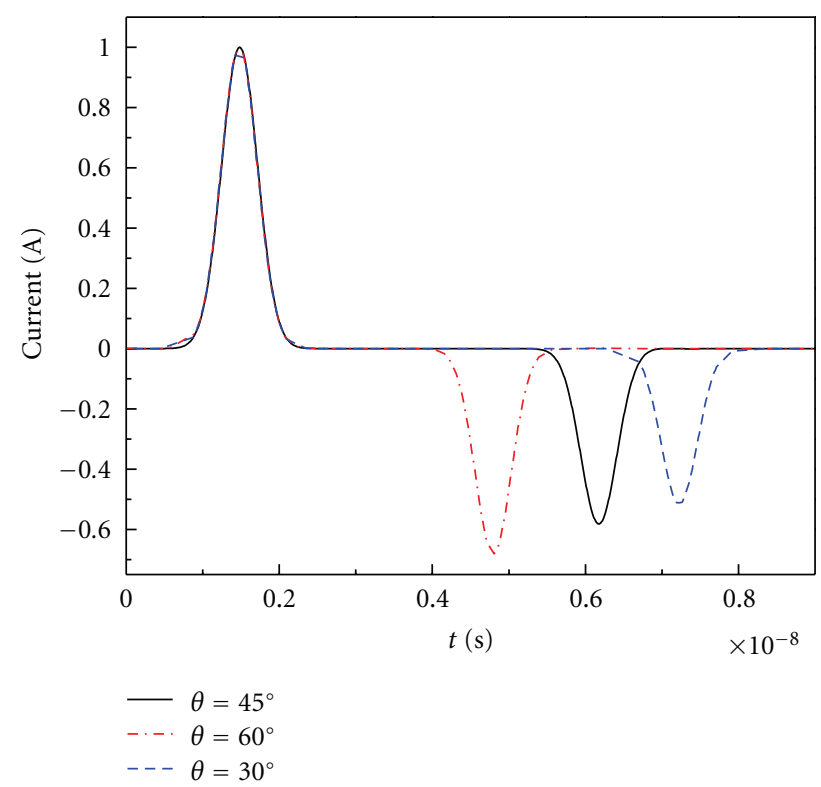

FIGURE 6: The incident and reflected wave on the height of thin wire with different incident angles.

Substituting (17) into (16), we have

$$
\begin{aligned}
I_{m, n} \kappa_{m, m}= & -A_{m, n}+2 A_{m, n-1}-A_{m, n-2}+(\Delta t)^{2} F_{m, n-1} \\
& +\left(\frac{c \Delta t}{\Delta x}\right)^{2}\left[A_{m+1, n-1}-2 A_{m, n-1}+A_{m-1, n-1}\right]
\end{aligned}
$$




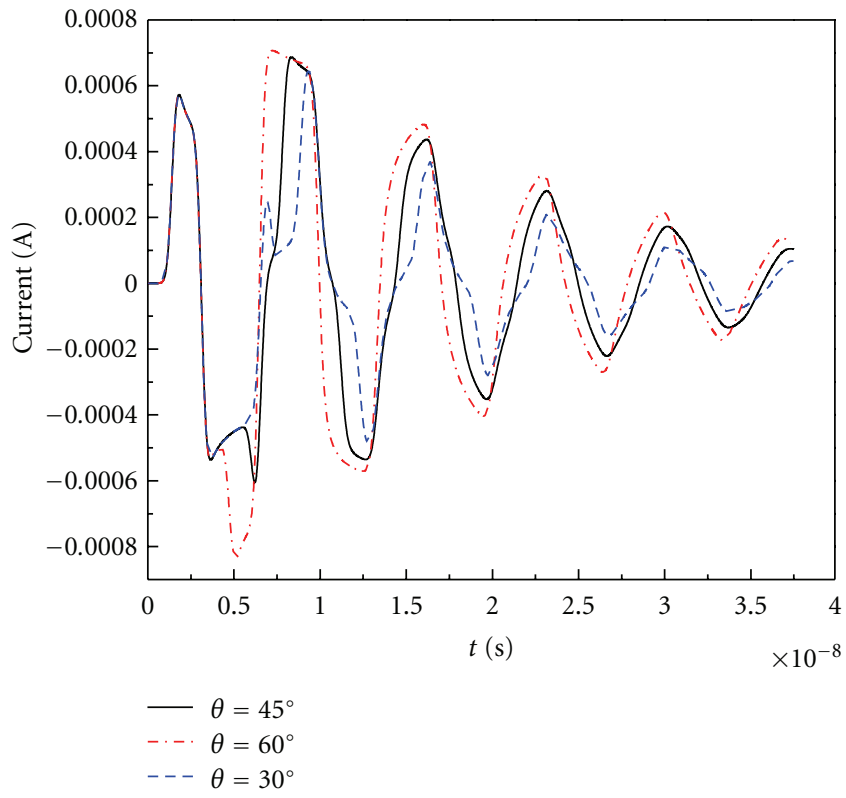

FIgURE 7: The transient current induced at midpoint of thin wire above half-space with different incident angles.

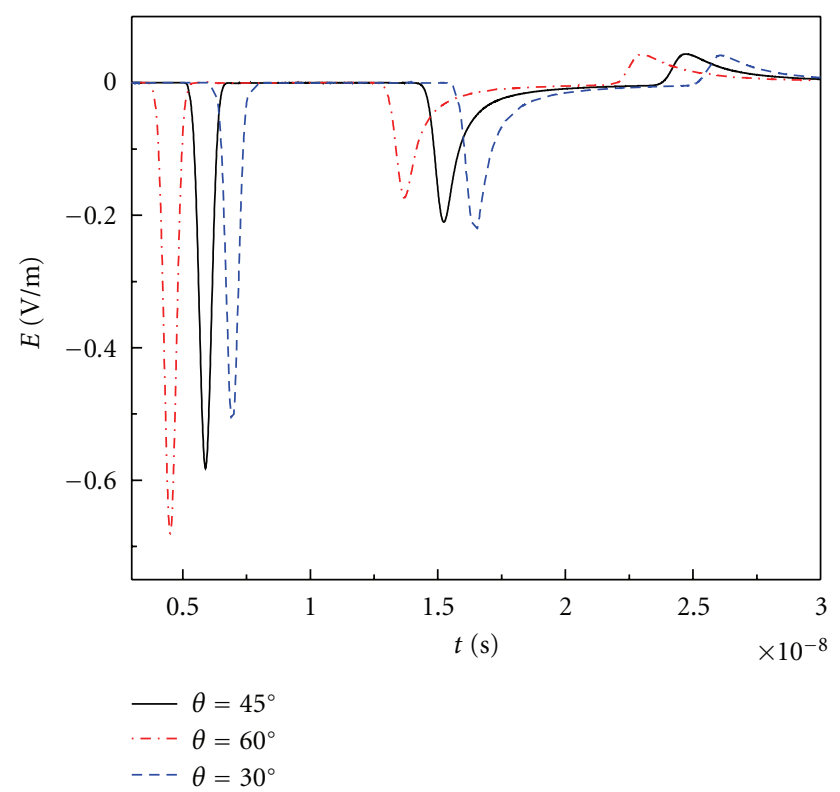

FIGURE 8: The reflected wave on the height of thin wire located above the half-space with different incident angle.

where

$$
\begin{gathered}
A_{m, n}=I_{m}\left(t_{n}\right) \kappa_{m, m}+\sum_{\substack{k=1 \\
k \neq m}}^{N} I_{k}\left(t_{n}-\frac{\left|x_{m}-x_{k}\right|}{c}\right) \kappa_{m, k}, \\
A_{m, n}=\sum_{\substack{k=1 \\
k \neq m}}^{N} I_{k}\left(t_{n}-\frac{\left|x_{m}-x_{k}\right|}{c}\right) \kappa_{m, k}, \\
I_{m, n} \equiv I_{m}\left(t_{n}\right),
\end{gathered}
$$

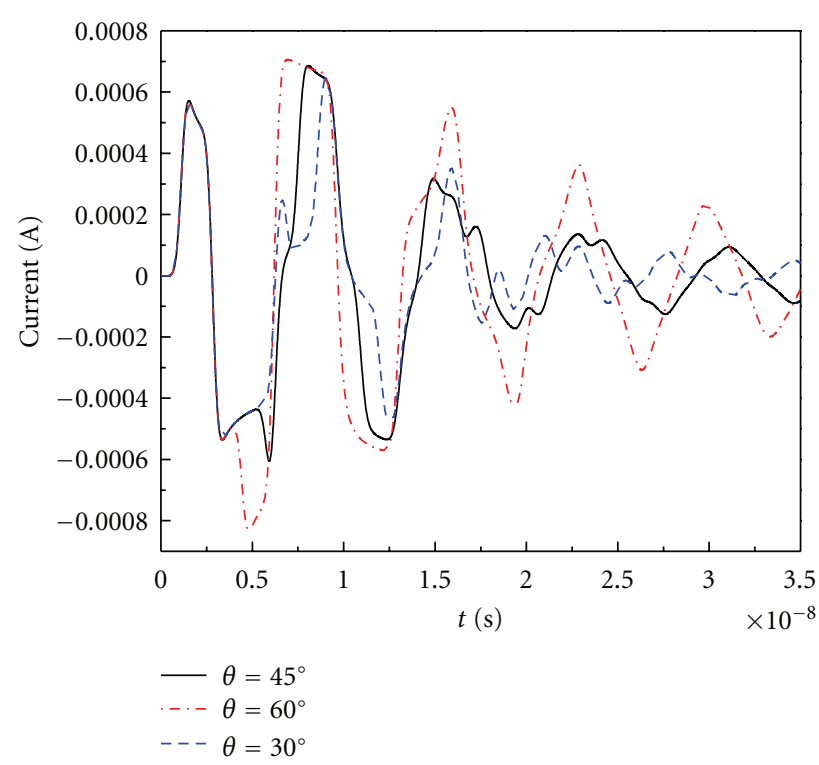

FIGURE 9: The current induced at midpoint of thin wire with different incident angle.

where $\kappa_{m, k}$ is impedance coefficient matrix. The algorithm may be started by assuming $I_{m, 0}=I_{m, 1}=0$ and calculating $I_{m, 2}$ using (18). Once we obtain $I_{m, 2}$, coupled with the knowledge of $I_{m, 0}$ and $I_{m, 1}$, we proceed to calculate $I_{m, 3}$ again using (18). This procedure can be continued to calculate currents at successive time instants $t_{4}, t_{5}, \ldots$ until the transient currents die down.

\section{Numerical Results}

In this section, numerical examples are given to verify the accuracy of the presented algorithm. Then, the transient responses of the straight thin wires above different layered half-spaces are analyzed. In all examples of this section, the length of thin wire is $l=1 \mathrm{~m}$, and the time step is the same in both TDIE and FDTD methods, which make it convenient for calculation in hybrid method.

Figure 5 gives the transient current (solid line) induced at the midpoint of this thin wire, which is at the height of $h=0.25 \mathrm{~m}$ above the interface. The underlying halfspace is homogeneous with relative permittivity $\varepsilon_{r}=10$, and the Gaussian pulse $\boldsymbol{E}=E_{0} \exp \left(-g^{2}\left(t-t_{0}\right)^{2}\right.$ ) (where $\left.E_{0}=1 \mathrm{~V} / \mathrm{m}, g=4.0 \times 10^{9} \mathrm{~s}^{-1}, t_{0}=1.2 \times 10^{-9} \mathrm{~s}\right)$ is incident perpendicularly to the interface of the half-space $\left(\varphi=90^{\circ}\right.$, $\left.\theta=0^{\circ}\right)$. The polarizing direction of the electric field is along $y$ axis. The results of [5] (dot) are also given for comparison in Figure 5. It is obvious that the results obtained by hybrid method are in good agreement with the results of [5]. This illustrates the correctness of the presented scheme.

The second example gives the comparison between directly incident wave and the reflected wave by the halfspace. The radius $a=0.002 \mathrm{~m}$, and the height $h=0.5 \mathrm{~m}$. It is illuminated by a Gaussian pulse $\left(E_{0}=1 \mathrm{~V} / \mathrm{m}, g=2.5 \times\right.$ $\left.\left.10^{9} \mathrm{~s}^{-1}\right), t_{0}=1.2 \times 10^{-9} \mathrm{~s}\right)$. The half-space is a nonmagnetic 


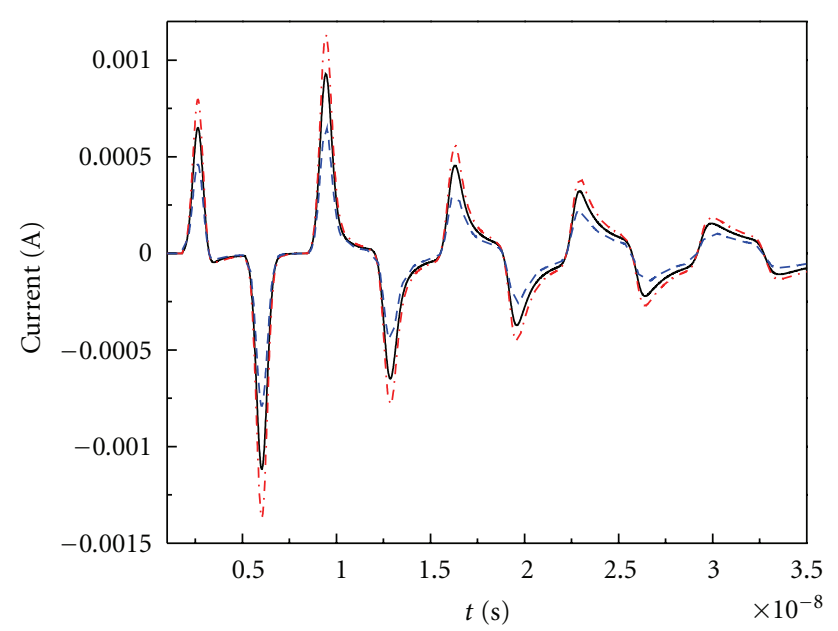

$\begin{aligned}-\varphi & =45^{\circ} \\ -\varphi & =60^{\circ} \\ --\varphi & =30^{\circ}\end{aligned}$

(a)

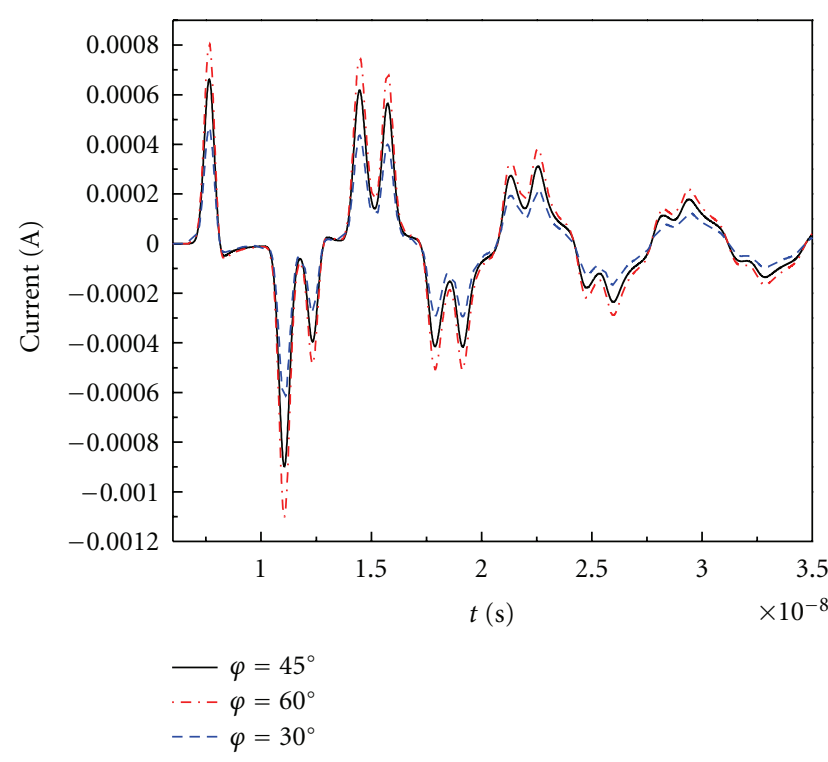

(b)

FIgURE 10: The current induced at midpoint of thin wire with different incident angle.

medium with $\varepsilon_{r}=4$ and $\sigma=10^{-5} \mathrm{~s} / \mathrm{m}$. Figure 6 presents the incident wave and reflected wave when $\varphi=90^{\circ}$ and the angle between the incident plane and the surface of the half-space is changed. The dash line, solid line, and dash dot line represent incident angle $\theta=30^{\circ}, \theta=45^{\circ}$, and $\theta=60^{\circ}$, respectively. It can be seen from Figure 6 that the amplitude of reflected wave is less than the original incident wave. There is also obvious time delay compared with the original incident wave.

Figure 7 plots the induced currents at the center of thin wire varying with time. The meaning of dashed line, solid line, and dash-dot line is same as above. As shown in the figure, the transient responses of different incident angles are the same at the early time and afterwards have obvious difference.

Figure 8 gives the reflected wave of the height of thin wire located above the layered half space with different incident angle. The reflected electric fields have multiple peaks, which is due to the presence of three interfaces and the interactions between the layers.

Then let us consider the transient response of a thin wire above layered half-space. As the configuration shown in Figure 1 exhibits, the thin wire is located at the height $h=0.5 \mathrm{~m}$ above the interface. The multilayer medium is composed as follows: the first layer is dry soil $\left(\varepsilon_{r}=4\right.$, $\sigma=10^{-5} \mathrm{~s} / \mathrm{m}$, thickness $\left.d_{1}=1.0 \mathrm{~m}\right)$; the second layer is wet soil $\left(\varepsilon_{r}=10, \sigma=10^{-3} \mathrm{~s} / \mathrm{m}\right.$, thickness $\left.d_{2}=1.0 \mathrm{~m}\right)$; the third layer is ground water $\left(\varepsilon_{r}=81, \sigma=10^{-3} \mathrm{~s} / \mathrm{m}\right)$. The length and the radius of the thin wire are the same as in the above example. The incident wave is the Guassain pulse $\left(E_{0}=1 \mathrm{~V} / \mathrm{m}, g=2.5 \times 10^{9} \mathrm{~s}^{-1}, t_{0}=0.86 \times 10^{-9} \mathrm{~s}\right)$, which is oblique to the layered interface. Figure 7 gives the reflected wave of the height of thin wire located above the layered half-space with different incident angles. The reflected electric fields have multiple peaks, which is due to the presence of three interfaces and the interactions between the layers.

Figure 9 gives the transient currents induced at the center of thin wire with different incident angles. The meaning of dash line, solid line, and dash-dot line is the same as in the above example. As shown in this figure, the transient responses are thesame at the early time and show obvious difference at latter time. This is because the effect of the interfaces reaches the wire at the latter time.

The last example is about the transient responses of thin wire above layered half-space when the incident angle $\varphi$ and $\theta$ are changed. The background and the location of wire are the same as in the above example. Figure 10(a) plots the induced current at the center of thin wire when the incident wave vector is at the plane which is perpendicular to the interface of the layered half-space $\left(\theta=90^{\circ}\right)$. The dash line, solid line, and dash dot line represent incident angle $\varphi=30^{\circ}$, $\varphi=45^{\circ}$, and $\varphi=60^{\circ}$, respectively. Figure $10(\mathrm{~b})$ plots the induced current at the center of thin wire when $\theta=45^{\circ}$. Also, the dash line, solid line, and dash dot line represent incident angles $\varphi=30^{\circ}, \varphi=45^{\circ}$, and $\varphi=60^{\circ}$, respectively. We can see from these two figures, the amplitudes of the current are different and the fluctuation time periods are obviously different when different incidence angles are used.

\section{Conclusion}

The TDIE/FDTD hybrid method is efficient for commutating the transient responses of thin wire above layered halfspace in the case of the EM oblique incident wave. Onedimensional TDIE is applied to study the above wire structure whereas one-dimensional modified FDTD method is used to get reflected field of the layered half-space. This can save computing time and memory, so the presented algorithm consumes less memory, offers high speed of computing, and is a highly efficient numerical solution. 


\section{Acknowledgment}

This work was supported by the National Natural Scientific Foundation of China under Grant 60871071 and the Fundamental Research Funds for the Central Universities.

\section{References}

[1] S. M. Rao and T. K. Shanker, Time Domain Electromagnetics, Academic Press, 1999.

[2] S. M. Rao, T. K. Sarkar, and S. A. Dianat, "The application of the conjugate gradient method to the solution of transient electromagnetic scattering from thin wires," Radio Science, vol. 19, no. 5, pp. 1319-1326, 1983.

[3] P. R. Barnes and F. M. Tesche, "On the direct calculation of a transient plane wave reflected from a finitely conducting half space," IEE Transactions on Electromagnetic Compatibility, vol. 33, no. 2, pp. 90-96, 1991.

[4] D. Poljak and V. Roje, "Time domain calculation of the parameters of thin wire antennas and scatterers in a half-space configuration," IEEE Proceedings-Microwaves, Antennas and Propagation, vol. 145, no. 1, pp. 57-63, 1998.

[5] D. Poljak, C. Y. Tham, and A. McCowen, "Transient response of nonlinearly loaded wires in a two media configuration," IEEE Transactions on Electromagnetic Compatibility, vol. 46, no. 1, pp. 121-125, 2004.

[6] M. H. Haddad, M. Ghaffari-Miab, and R. Faraji-Dana, "Transient analysis of thin-wire structures above a multilayer medium using complex-time Green's functions," IET Microwaves, Antennas and Propagation, vol. 4, no. 11, pp. 1937-1947, 2010.

[7] L. Li, L. Li, C. Gao, X. Gu, and Z. Zhao, "Numerical treatment for time domain integral equations of thin wire structures in half-space configuration," IEEE Transactions on Magnetics, vol. 44, no. 6, Article ID 4526938, pp. 774-777, 2008.

[8] L. Li, L.-J. Li, and C.-Z. Gao, "Direct time-domain method for analyzing scattering from thin wire structures in the dielectric half-space," Journal of North China Electric Power University, vol. 34, no. 6, 2005.

[9] T. C. Tong and A. Sankar, "Transient scattering from curved thin wires by the finite element method," IEEE Antennas and Propagation Society, vol. 12, no. 3, 1980.

[10] P. Parhami and R. Mittra, "Wire antennas over a lossy halfspace," IEEE Transactions on Antennas and Propagation, vol. AP-28, pp. 397-403, 1980.

[11] B. Wei, X. Y. Li, F. Wang, and D. B. Ge, "A finite difference time domain absorbing boundary condition for general frequencydispersive media," Acta Physica Sinica, vol. 57, no. 10, pp. 6283-6289, 2008.

[12] Y. N. Jiang and D. B. Ge, "New scheme for introducing an oblique incidence plane wave to layered media in finitedifference time-domain," Acta Physica Sinica, vol. 58, no. 9, pp. 6174-6178, 2009.

[13] K. S. Yee, "Numerical solution of initial boundary value problems involving Maxwell's equations in isotropic media," IEEE Transactions on Antennas and Propagation, vol. 14, pp. 302-307, 1966.

[14] A. Taflove and M. E. Brodwin, "Numerical solution of steady-state electromagnetic scattering problems using the time-dependent Maxwell's equations," IEEE Transactions on Microwave Theory and Techniques, vol. 23, no. 8, pp. 623-630, 1975.
[15] A. Taflove, "Application of the finite-difference time-domain method to sinusoidal steady-state electromagnetic penetration problems," IEEE Transactions on Electromagnetic Compatibility, vol. 22, no. 3, pp. 191-202, 1980.

[16] A. Taflove and S. C. Hagness, Computational Electrodynamics: The Finite-Difference Time-Domain Method, Artech House, Norwood, Mass, USA, 3rd edition, 2005.

[17] Z. H. Chen and Q. X. Chu, "FDTD modeling of arbitrary linear lumped networks using piecewise linear recursive convolution technique," Progress in Electromagnetics Research, vol. 73, pp. 327-341, 2007.

[18] M. Ali and S. Sanyal, "FDTD analysis of dipole antenna as EMI sensor," Progress in Electromagnetics Research, vol. 69, pp. 341$359,2007$.

[19] L. X. Yang, D. B. Ge, and B. Wei, "FDTD/TDPO hybrid approach for analysis of the EM scattering of combinative objects," Progress in Electromagnetics Research, vol. 76, pp. 275-284, 2007.

[20] Y. N. Jiang, D. B. Ge, and S. J. Ding, "Analysis of TF-SF boundary for 2D-FDTD with plane P-wave propagation in layered dispersive and lossy media," Progress in Electromagnetics Research, vol. 83, pp. 157-172, 2008.

[21] S. W. Yang, Y. K. Chen, and Z. P. Nie, "Simulation of time modulated linear antenna arrays using the FDTD method," Progress in Electromagnetics Research, vol. 98, pp. 175-190, 2009.

[22] Y. Q. Zhang and D. B. Ge, "A unified FDTD approach for electromagnetic analysis of dispersive objects," Progress in Electromagnetics Research, vol. 96, pp. 155-172, 2009.

[23] Y. Tretiakov and G. Pan, "Malvar wavelet based pocklington equation solutions to thin-wire antennas and scatters," Progress In Electromagnetics Research, vol. 47, pp. 123-133, 2000.

[24] K. Afrooz, A. Abdipour, A. Tavakoli, and M. Movahhedi, "Time domain analysis of active transmission line using FDTD technique (Application to microwave/MM-wave transistors)," Progress in Electromagnetics Research, vol. 77, pp. 309-328, 2007.

[25] M. Y. Wang, J. Xu, J. Wu et al., "FDTD study on wave propagation in layered structures with biaxial anisotropic metamaterials," Progress in Electromagnetics Research, vol. 81, pp. 253-265, 2008.

[26] Z. Huang, K. Demarest, and R. Plumb, "An FDTD/MoM hybrid techinique for modeling complex antennas in the presence of heterogeneous grounds," IEEE Transactions on Geoscience and Remote Sensing, vol. 37, no. 6, pp. 2692-2698, 1999.

[27] A. Monorchio, A. Rubio Bretones, R. Mittra, G. Manara, and R. Gómez Martín, "A hybrid time-domain technique that combines the finite element, finite difference and method of moment techniques to solve complex electromagnetic problems," IEEE Transactions on Antennas and Propagation, vol. 52, no. 10, pp. 2666-2674, 2004.

[28] F. Edelvik, "A new technique for accurate and stable modeling of arbitrarily oriented thin wires in the FDTD method," IEEE Transactions on Electromagnetic Compatibility, vol. 45, no. 2, pp. 416-423, 2003.

[29] H. Zhao and Z. Shen, "Weighted Laguerre polynomials-finite difference method for time-domain modeling of thin wire antennas in a loaded cavity," IEEE Antennas and Wireless Propagation Letters, vol. 8, Article ID 5286304, pp. 1131-1134, 2009.

[30] J. A. Kong, Electromagneitc Wave Theory, EMW Publishing, Higher Education Press, 2002. 

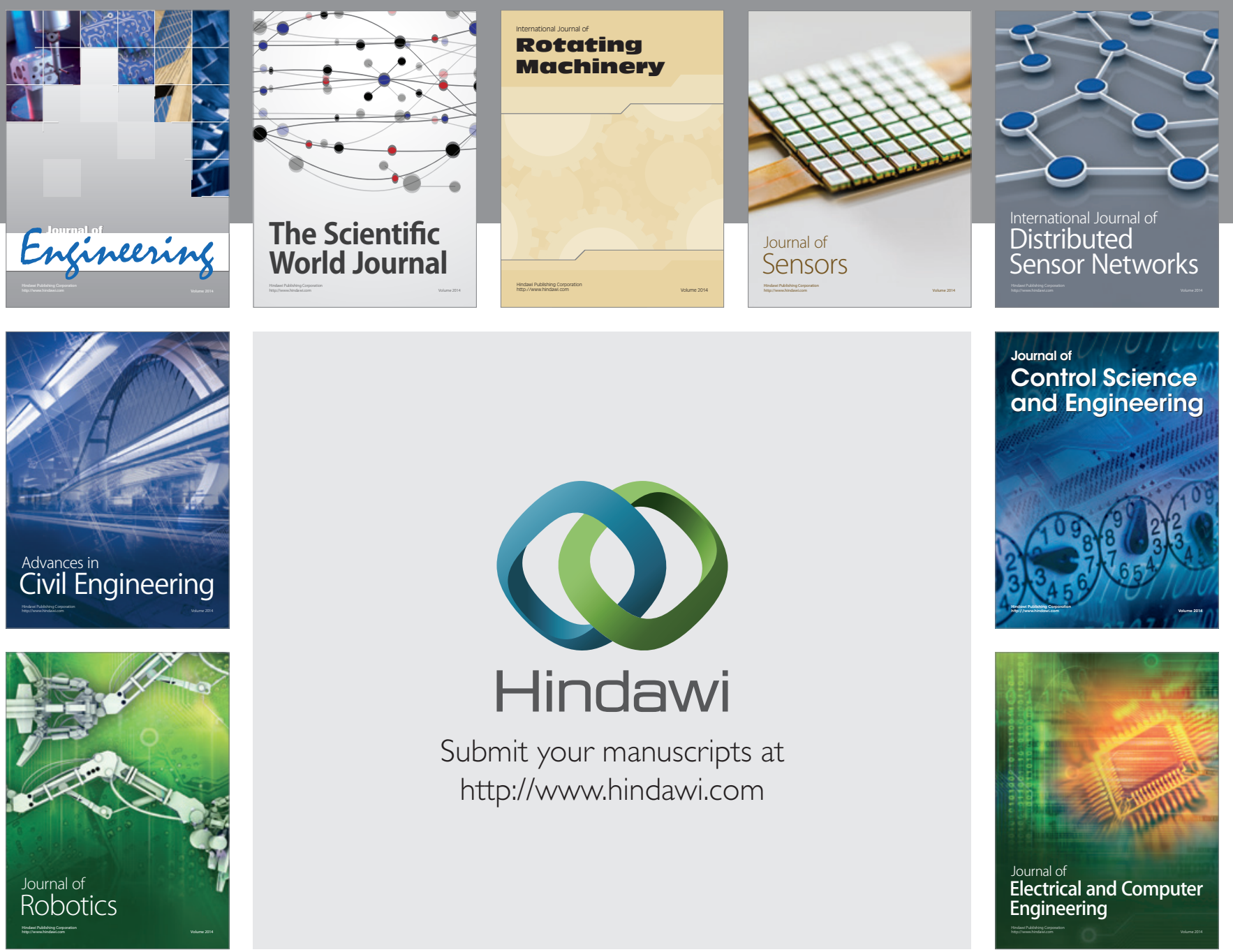

Submit your manuscripts at

http://www.hindawi.com
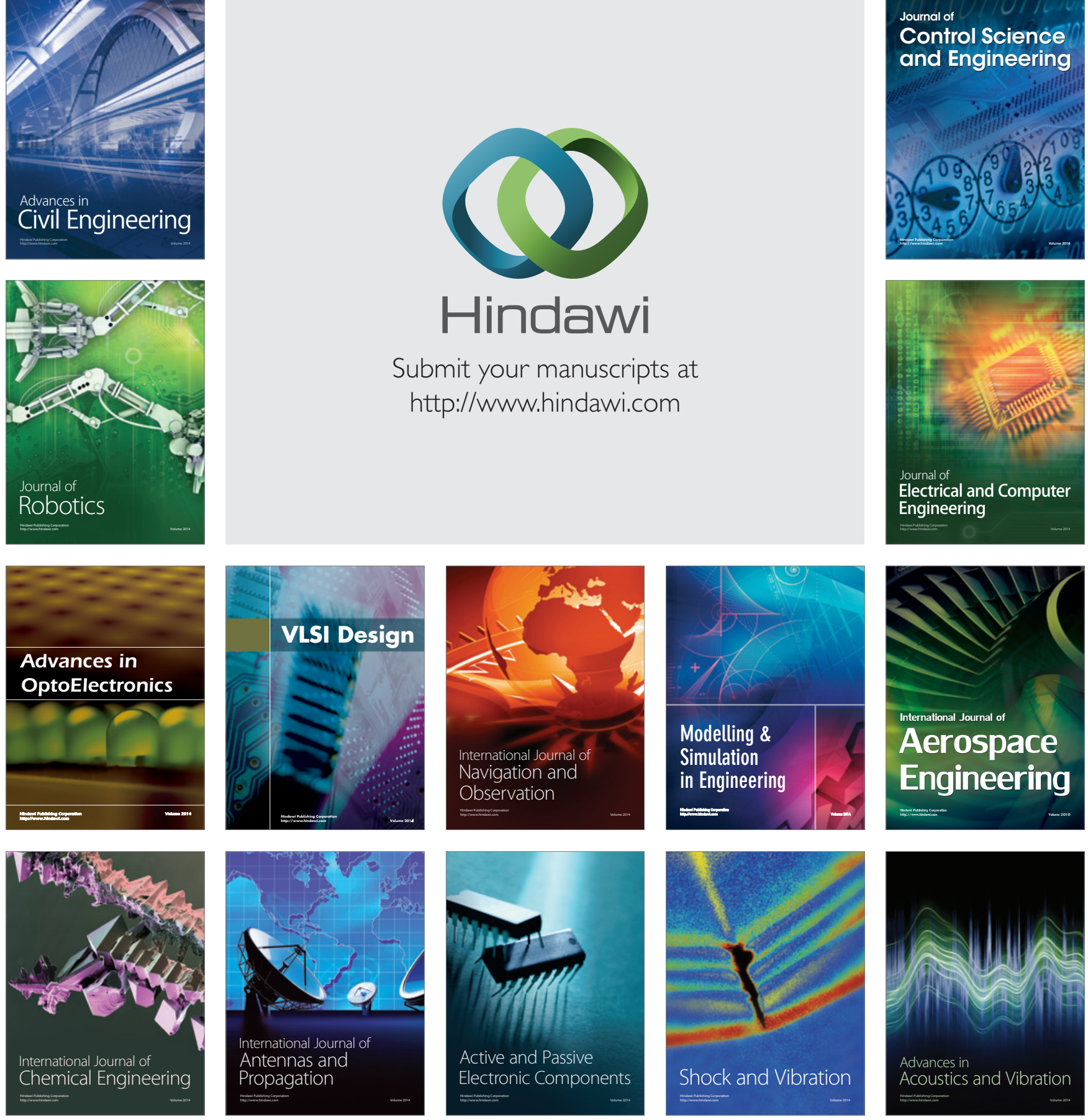